\title{
Dyspnea with a Slow-Growing Mass in the Breast
}

\author{
Misato Amenomori ${ }^{a}$ Noriho Sakamoto $^{a}$ Kazuto Ashizawa ${ }^{b}$

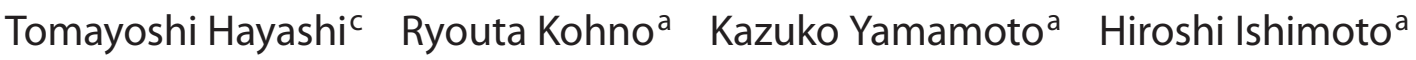 \\ Hiroshi Mukae $^{\text {a Shigeru Kohno }}{ }^{a}$ \\ aSecond Department of Internal Medicine, Nagasaki University School of Medicine, ${ }^{\mathrm{b}}$ Clinical Oncology Center, and \\ 'Department of Pathology, Nagasaki University Hospital, Nagasaki, Japan
}

A 48-year-old Japanese woman presented with a 6month history of increasing exertional dyspnea and dry cough. She denied fever, night sweats, or weight loss. She was a nonsmoker. Her medical history was unremarkable except for the fact that she had been aware of a mass in her left breast for $>2$ years.

On admission, her temperature was $36.7^{\circ} \mathrm{C}$, her pulse was regular at 70 b.p.m., and her respiratory rate was 16 breaths/min. Her lungs were clear on auscultation, and cardiac examination revealed no murmur or gallops. No edema or varicosities were seen in her lower extremities. A hard mass measuring up to $3 \mathrm{~cm}$ in diameter was palpable in her left breast. Oxygen saturation was $94 \%$ on room air. Arterial blood gases on room air were $\mathrm{pH} 7.404$, $\mathrm{PaCO}_{2} 5.5 \mathrm{kPa}(40.9 \mathrm{~mm} \mathrm{Hg}), \mathrm{PaO}_{2} 9.3 \mathrm{kPa}(69.4 \mathrm{~mm} \mathrm{Hg})$, and $\mathrm{A}-\mathrm{aDO}_{2} 4.2 \mathrm{kPa}(31.5 \mathrm{~mm} \mathrm{Hg})$. On laboratory examination, the complete blood count and basic chemistries were within the normal ranges. D-dimer was 2.5 (normal value $<1.0) \mu \mathrm{g} / \mathrm{ml}$, and the thrombin-antithrombin III complex was 7.0 (normal value $\leq 3.0$ ) $\mathrm{ng} / \mathrm{ml}$. Fibrinogen and fibrinogen degradation products were within their normal ranges. The results of pulmonary function tests were: VC 1.86 liters, \%VC 69.1\%, $\mathrm{FEV}_{1.0} 1.88$ liters, $\mathrm{FEV}_{1.0} \% 100.0 \%$, DLCO $8.37 \mathrm{ml} / \mathrm{mm} / \mathrm{mm} \mathrm{Hg}$, and \%DLCO 51.4\%. Echo- and electrocardiograms disclosed no findings of cardiac failure or pulmonary hypertension.

\section{KARGER}

Fax +41613061234 E-Mail karger@karger.ch www.karger.com
(C) 2009 S. Karger AG, Basel

0025-7931/10/0794-0346\$26.00/0

Accessible online at:

www.karger.com/res
Chest radiography showed multiple nodular opacities in both peripheral lung fields. Thin-section CT of the chest revealed multiple tiny nodules and centrilobular branching opacities in the subpleural regions of both lung fields (fig. 1a). Wedge-shaped or irregularly shaped nodular opacities suggesting pulmonary infarction were also seen in subpleural distribution (fig. 1b). On contrastenhanced CT of the mediastinal window setting, a wellenhanced mass in the left breast, up to $3 \mathrm{~cm}$ in diameter, was seen (fig. 1c). No mediastinal or hilar lymphadenopathy was identified. There were no pleural effusions.

The patient underwent bronchoscopy that showed normal endobronchial mucosa. Bronchoalveolar lavage was not diagnostic; no organisms or malignant cells were seen on lavage fluid stains, and the fluid was sterile.

Transbronchial lung biopsy (TBLB) was performed from right $\mathrm{B}^{4}$. Pathologically, scattered thrombi involving small pulmonary arteries and arterioles were seen.

Simultaneously, a ventilation-perfusion lung scintigram was performed. Multiple minute defects in the peripheral lung fields just under the pleura were seen on the perfusion scintigram, while the ventilation scintigram showed normal ventilation.

In view of these findings, what is your diagnosis?
Noriho Sakamoto, $\mathrm{MD}, \mathrm{PhD}$

Second Department of Internal Medicine

Nagasaki University School of Medicine

1-7-1 Sakamoto, Nagasaki 852-8501 (Japan)

Tel. +8195819 7273, Fax +8195849 7285, E-Mail nsakamot@ nagasaki-u.ac.jp 
Fig. 1. a, b Thin-section CT images of the chest obtained using 1-mm collimation: lung window settings disclose multiple tiny nodules and centrilobular branching opacities in the subpleural regions of both lung fields (arrows). Wedge-shaped or irregularly shaped nodular opacities are also seen in a subpleural distribution (arrowhead). c Contrast-enhanced CT image of the chest obtained using 7-mm collimation: mediastinal window setting shows a well-enhanced mass in the left breast, measuring up to $3 \mathrm{~cm}$ in diameter (arrow). No mediastinal or hilar lymphadenopathy is seen. There are no pleural effusions.
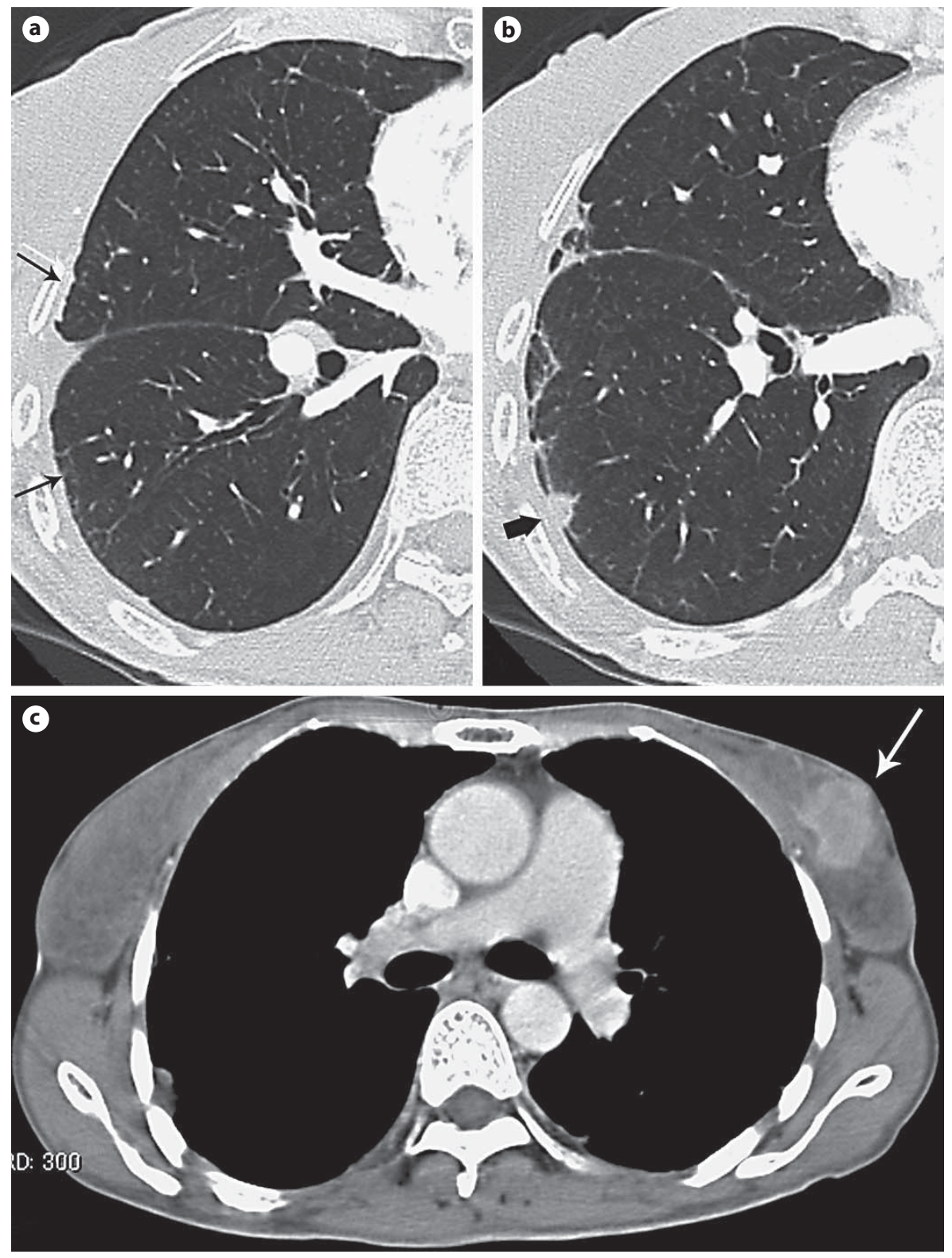


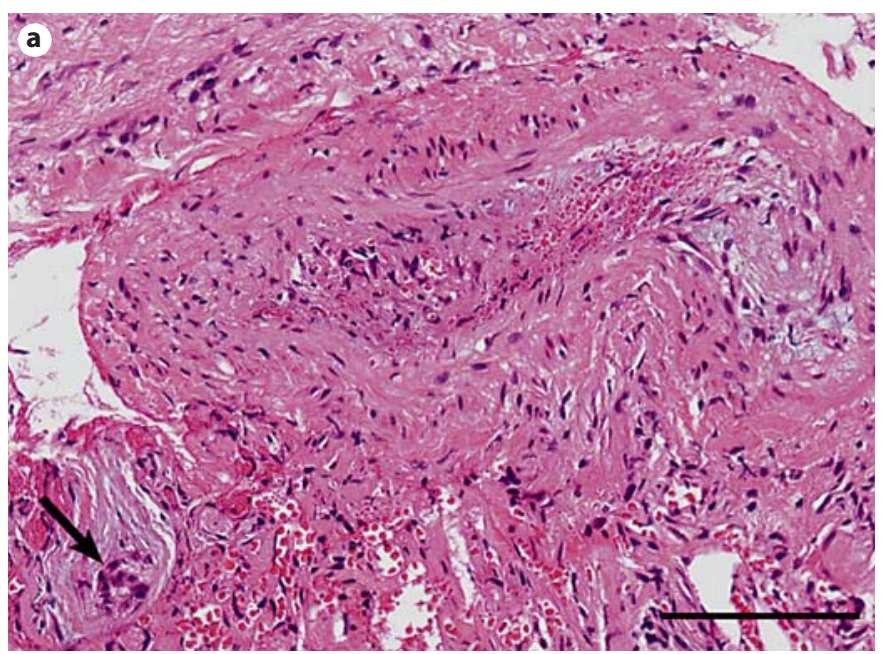

Fig. 2. Serial sections of the TBLB specimens from right $B^{4}$ stained with hematoxylin-eosin (HE), elastic van Gieson (EVG) and estrogen receptor, respectively. a The small muscular arteries are obstructed with thrombi and intimal thickening. In the thickened intima, entrapped atypical cells are observed (arrow; HE). b EVG staining highlights two arteries with elastic laminae, which are obstructed by thrombi and intimal thickening. c Immunohistochemical staining for estrogen receptor showing weakly positive staining in the nuclei of atypical cells entrapped in the thrombus and intimal thickening (arrowheads). Scale bar $=100 \mu \mathrm{m}$.

In addition to the multiple thrombi in small pulmonary arteries, there were abnormal cells in the arterial lumen. Therefore, the existence of underlying malignancy was suspected, but the primary lesion was unknown at that time. Therefore, a core needle biopsy of the breast mass was performed, and the diagnosis of invasive ductal carcinoma was made. Immunohistochemically, strong reactivity for estrogen and progesterone receptors was seen in the breast and lung tissue. Reevaluating the lung specimen in light of this evidence, the small arteries and arterioles were obstructed with organized thrombi and intima proliferation (fig. 2). Findings of recanalization were also seen.
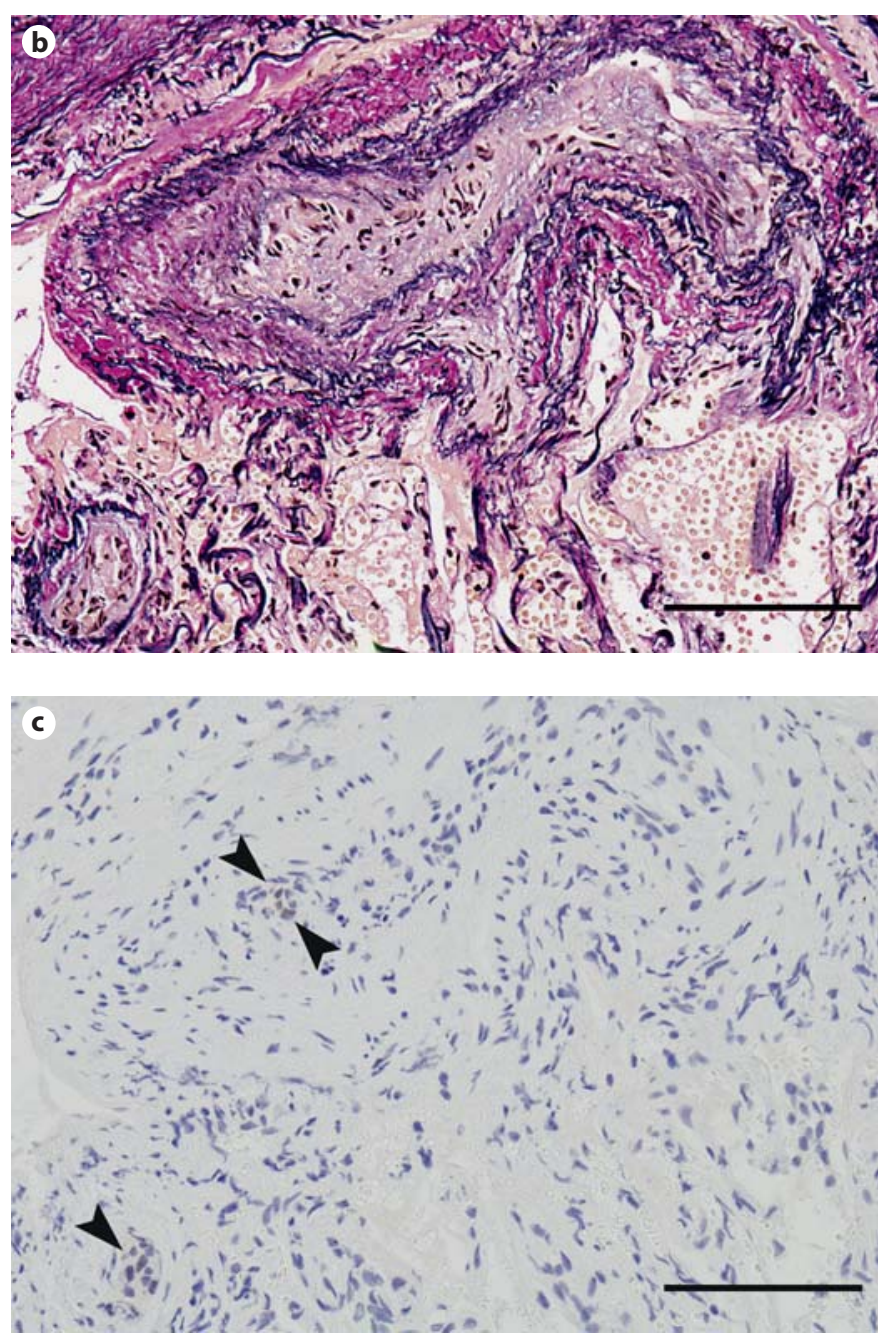

On the 19th day after admission, anticoagulant therapy with warfarin sodium was started. The patient was scheduled to have anticancer or hormone therapy within a short time, but she refused to receive further treatment. Thus, she never returned to our hospital, and we are not aware of her outcome at present.

Pulmonary tumor thrombotic microangiopathy (PTTM) is a rare type of pulmonary tumor embolism with tumor-related microangiopathic lesions in small pulmonary arteries and arterioles [1]. PTTM is detected in $0.9-3.3 \%$ autopsies of patients with malignant tumors $[1,2]$. Although PTTM is relatively well discussed in a number of case reports, the diagnosis of PTTM was made overwhelmingly on autopsy [3-7], and cases of PTTM 
with antemortem diagnosis, including our present case, are very rare $[8,9]$.

Acute or subacute progressive dyspnea with pulmonary hypertension is a typical initial clinical presentation, and acute heart failure followed by severe pulmonary hypertension is the main cause of death $[6,8-11]$. The current case presented with exertional dyspnea that had been progressive over a 6 -month period. Slight hypoxemia was also seen, but findings of cardiac failure or pulmonary hypertension were not apparent. This may be because disease extent and progression were mild and very slow, i.e. she visited our hospital before her general condition had become critical.

Because PTTM occurs mainly in small arteries and arterioles, cases showing minute or almost normal radiographic findings are occasionally reported $[3,6]$. Thinsection CT findings of PTTM have been described in only 2 case reports in the English literature previously; they had multiple small nodules and centrilobular branching opacities located predominantly just under the pleura $[9,10]$. In cases of pulmonary hypertension, prominent pulmonary arteries are commonly detected $[6,9]$.

Histologically, PTTM is characterized by widespread tumor emboli associated with fibrocellular intimal proliferation and thrombus formation in the small arteries and arterioles of the lungs [1]. The pathogenetic events start with metastases and adherence of small clusters of tumor cells to the pulmonary arterial system and adherence to the vascular endothelium at a microscopic level. Tumor emboli do not directly occlude affected vessels, but they induce local activation of coagulation, release of inflammatory mediators, and fibrocellular intimal proliferation, leading to stenosis or occlusion of the vessel [1, $3,5]$. From this point of view, PTTM should be distinguished from tumor embolism [9], and to clarify the diagnosis, histological investigation is indispensable.

In case of PTTM with unknown primary malignant lesion, immunohistochemical staining for CK7, CK20,
TTF-1, and hormone receptors has been reported to be useful in providing a clue regarding the nature of the primary lesion. It is also beneficial to confirm the relationship between the pulmonary findings and a known malignancy $[12,13]$. In the present case, pulmonary embolism appeared to be highly likely from the radiological findings, but immunohistochemical study of the tissue led to a prompt and accurate diagnosis.

In cases diagnosed antemortem, the diagnoses were made on the basis of surgical lung specimens or TBLB $[8$, 9]. Although TBLB is limited and not all abnormalities are present in the tissues obtained, TBLB can provide a clue to diagnose PTTM. Recently, pulmonary microvascular cytology using a pulmonary artery catheter has also been reported to be beneficial [14].

The standard treatment for PTTM has not yet been established [6]. The other previously reported case diagnosed during life was successfully treated with aspirin, warfarin potassium, dexamethasone, and oral anticancer drug therapy [9], though it is not clear which had the best effect on PTTM. Coagulation tends to be activated in patients with cancer [15], and similar to the treatment of cancer-associated venous thrombosis, low-molecularweight heparin, which provides a good predictable anticoagulant response [16], is generally recommended in order to improve patient survival [17]. Thus, anticoagulant therapy may be effective in treating PTTM.

In summary, although PTTM is rare, it should be considered in the differential diagnosis of progressive dyspnea, even if malignancy is not apparent at the time of presentation. It is important to emphasize that TBLB can provide clues regarding the diagnosis of PTTM.

\section{Key Words \\ Breast cancer $\cdot$ Pulmonary tumor $\cdot$ Thrombotic microangiopathy $\cdot$ Transbronchial lung biopsy}

\section{References}

Dyspnea with a Slow-Growing Mass in the Breast
1 von Herbay A, Illes A, Waldherr R, Otto H: Pulmonary tumor thrombotic microangiopathy with pulmonary hypertension. Cancer 1990;66:587-592.

2 Nakamura H, Adachi $H$, Sudoh A, Yagyu H, Kishi K, Oh-ishi S, Kusama H, Hashimoto T, Matsuoka T: Subacute cor pulmonale due to tumor embolism. Intern Med 2004;43:420 422.
3 Pinckard JK, Wick MR: Tumor-related thrombotic pulmonary microangiopathy: review of pathologic findings and pathophysiologic mechanisms. Ann Diagn Pathol 2000;4:154-157.

4 Chinen K, Kazumoto T, Ohkura Y, Matsubara O, Tsuchiya E: Pulmonary tumor thrombotic microangiopathy caused by a gastric carcinoma expressing vascular endothelial growth factor and tissue factor. Pathol Int 2005;55:27-31. 
5 Yao DX, Flieder DB, Hoda SA: Pulmonary tumor thrombotic microangiopathy: an often missed antemortem diagnosis. Arch Pathol Lab Med 2001;125:304-305.

6 Keenan NG, Nicholson AG, Oldershaw PJ: Fatal acute pulmonary hypertension caused by pulmonary tumour thrombotic microangiopathy. Int J Cardiol 2008;124:e11-e13.

7 Malani AK, Gupta C, Kutty AV, Betlej T: Pulmonary tumor thrombotic microangiopathy from metastatic gallbladder carcinoma: an unusual cause of severe pulmonary hypertension. Dig Dis Sci 2007;52:555-557.

8 Noguchi S, Imanaga T, Shimizu M, Nakano T, Miyazaki N: A case of pulmonary tumor thrombotic microangiopathy diagnosed by transbronchial lung biopsy (in Japnese). Nihon Kokyuki Gakkai Zasshi 2008;47:493496.
9 Miyano S, Izumi S, Takeda Y, Tokuhara M, Mochizuki M, Matsubara O, Kuwata H, Kobayashi N, Kudo K.: Pulmonary tumor thrombotic microangiopathy. J Clin Oncol 2007;25:597-599.

10 Franquet T, Gimenez A, Prats R, RodriguezArias JM, Rodriguez C: Thrombotic microangiopathy of pulmonary tumors: a vascular cause of tree-in-bud pattern on CT. AJR Am J Roentgenol 2002;179:897-899.

11 Sato Y, Marutsuka K, Asada Y, Yamada M, Setoguchi T, Sumiyoshi A: Pulmonary tumor thrombotic microangiopathy. Pathol Int 1995;45:436-440.

12 Cai YC, Banner B, Glickman J, Odze RD: Cytokeratin 7 and 20 and thyroid transcription factor 1 can help distinguish pulmonary from gastrointestinal carcinoid and pancreatic endocrine tumors. Hum Pathol 2001;32: 1087-1093.
$13 \mathrm{Su}$ YC, Hsu YC, Chai CY: Role of TTF-1, CK20, and CK7 immunohistochemistry for diagnosis of primary and secondary lung adenocarcinoma. Kaohsiung J Med Sci 2006; 22:14-19.

14 Masson RG, Ruggieri J: Pulmonary microvascular cytology. A new diagnostic application of the pulmonary artery catheter. Chest 1985;88:908-914

15 Pruemer J: Prevalence, causes, and impact of cancer-associated thrombosis. Am J Health Syst Pharm 2005;62:S4-S6.

16 Kroegel C: Advances in the diagnosis and treatment of pulmonary embolism. Pulmonary embolism - how can you mend a broken clot? Respiration 2001;70:4-6.

17 Behranwala KA, Williamson RC: Cancer-associated venous thrombosis in the surgical setting. Ann Surg 2009;249:366-375. 